\title{
Vitamin D during pregnancy: A strong player in a battle for two
}

\author{
Adina Ghemigian ${ }^{1,2}$, Andra Cocolos ${ }^{1,2}$, Nicoleta Dumitru ${ }^{1,2}$, Eugenia Petrova ${ }^{1}$, \\ Ana Valea ${ }^{3,4}$, Mara Carsote ${ }^{1,2}$ \\ 1"Carol Davila" University of Medicine and Pharmacy Bucharest, Romania \\ 2"C.I. Parhon" National Institute of Endocrinology, Bucharest, Romania \\ 3"Iuliu Hatieganu" University of Medicine and Pharmacy, Cluj-Napoca, Romania \\ ${ }^{4}$ Clinical County Hospital, Cluj-Napoca, Romania
}

\begin{abstract}
Vitamin D represents a complex endocrine system with multifunctional levels of action, including during pregnancy. This is a short review of literature based on PubMed research. Vitamin D is linked to numerous divergent normal and abnormal functions of the human body. This becomes crucial during pregnancy. Specific metabolism during this period of time includes the role of placenta as provider of additional calcitriol resources. The excess of calcitriol is sustained by adequate levels of maternal 25-hydroxyvitamin D. Different conditions as gestational diabetes have been linked to maternal hypovitaminosis D. Vitamin D also prepares neonates immune system. Vitamin D represents a strong player during pregnancy from mothers and child's as well point of view.
\end{abstract}

Keywords: vitamin D, pregnancy, calcitriol

\section{INTRODUCTION}

Vitamin D represents a complex a complex endocrine system with multifunctional display both in women and men, including special periods of time like pregnancy. We aim to introduce a brief review of dynamic approach regarding vitamin D during pregnancy and its implications for the foetal health. The ideas introduced in this paper are focused on 10 major points. The short research of literature is based on data published on PubMed.

\section{GENERAL DATA}

\section{Vitamin D endocrine system is linked to nu-} merous divergent normal and abnormal functions of the human body

Vitamin D is provided by sun - associated skin synthesis and also by intestinal absorption, including from supplements (1). Its activation is finalised by generating calcitriol both in kidney and non-re- nal tissues as monocytes - macrophages system, placenta, etc (1). 1,25 - dihydroxyvitamin D is responsible for calcium homeostasis; muscle and bone good health; modulation of blood pressure; cardiovascular system interplay; communication between fat, bone, brain; as well as neuro- and immune- modulation, and, also regulation of cell biology (growth, differentiation etc.) (1).

\section{Vitamin D becomes crucial during pregnancy}

Probably, nowhere else but during pregnancy, vitamin $\mathrm{D}$ is essential not only for mother but also for the foetus from a dual point of view: immune-modulation and future skeletal health (2). Differences are major between pregnancy and non-pregnancy status since dynamic changes of mother and developing foetus are registered (2). The best indicator of vitamin D status remains the pro-hormone 25OHD (25-hydroxyvitamin D) (2). The conversion of vitamin D3 from skin or intestine to 25-hydroxyvitamin D through 25-hydroxilation is similar as seen outside the pregnancy (2). 


\section{Specific metabolism during pregnancy: The role of placenta}

Initially, the role of vitamin D is to avoid foetal rejection and its necessary progressively increases but, via a specific pathway, since the $12^{\text {ve }}$ week its concentration dramatically increases up to levels two to three times higher than non-pregnancy levels because calcitriol is needed to prepare the mother and foetus skeleton (3). Placenta becomes the provider of additional amount of calcitriol due to its enzymatic activity (3).

\section{The maternal sources of vitamin $D$}

The excess of calcitriol is sustained by adequate levels of maternal 25-hydroxyvitamin D (4). This major source is for both immune-modulator and calcium regulating hormone (4). Barker hypothesis is related to adult-onset diseases caused by peri-natal nutritional (like asthma, multiple sclerosis) and vitamin D is one of the elements (4). The target of $25 \mathrm{OHD}$ should not be only the well known level of $30 \mathrm{ng} / \mathrm{mL}$, but maybe higher, for instance up to $40 \mathrm{ng} / \mathrm{mL}$, as considered by some societies (2).

\section{Low levels of $25 \mathrm{OHD}$}

Low maternal 25OHD is associated with developing a higher risk for infertility parameters, bacterial vaginosis, and, also, a need for primary caesarean section (5). A 2017 meta-analysis included 18 longitudinal studies and analyzed the potential correlation with spontaneous abortion and the risk of stillbirth in cases with hypovitaminosis D (5). Also, gestational diabetes, hypertensive disorders, and other complications of birth have been linked to the same condition (1).

\section{The role of calcium}

During pregnancy there are changes of intracellular free calcium concentration at the level of red blood cells, platelets, and immune cells (6). The increase of intracellular calcium at the level of endothelial cells induces the production of vasodilator substances such as nitric oxide and prostacyclin which are modulated by vitamin D and further connected to the risk of developing high blood pressure in normal pregnancy or in eclampsia (6).

\section{Vitamin D prepares neonates immune system}

Vitamin D supplementation modifies neonate's immune system and this is more equipped to pro- tect the host against infections, thus offering a protection against asthma and infections (7). Maternal hypovitaminosis D may cause a higher risk of asthma during early childhood (7).

8. Vitamin D as part of epigenetic mechanisms

Providing an adequate vitamin D supplementation influences transcriptome and epigenetic alterations in children through DNA methylation in genes (1). These processes include antigen processing, anti-inflammation mechanisms, and control of cell proliferation, modulation of neurogenesis and transmission of nerve impulse (1).

\section{Vitamin D and small for gestational age}

First systematic review with only prospective cohorts regarding vitamin D and small for gestational age correlation has substantial heterogeneity as results (8). 13 cohort studies were included, a total of 28285 individuals from 7 countries (8). Odds ratio for babies born small for gestational age is 1.588 (95\% CI $1.138-2.216 ; \mathrm{p}<0.01)$ from mothers with hypovitaminosis D (8). The prevalence of vitamin D deficiency during pregnancy is 13.2-77.3\% (8).

\section{Pregnancy and lactation associated osteo-} porosis

This is a severe type of premenopausal osteoporosis diagnosed during last trimester and/or immediately after birth, and potentially linked to insufficient vitamin D levels (9). Is represents a very rare clinical entity, including vertebral fractures much more frequent than hip fractures (9). Typically the onset is acute back pain (9). The therapeutically options are limited, but vitamin D and calcium supplements are recommended (9). A higher risk is described in populations with an increased prevalence of hypovitaminosis D (10).

\section{CONCLUSION}

By these 10 arguments, vitamin D represents a strong player during pregnancy from mothers and child's as well point of view.

Conflict of interest: none declared Financial support: none declared

\section{REFERENCES}

1. Kiely M., Hemmingway A., O'Callaghan K.M. Vitamin D in pregnancy: Current perspectives and future directions. Ther $A d v$ Musculoskelet Dis. 2017 Jun; 9(6):145-154.

2. Hollis B.W., Wagner C.L. New insights into the vitamin D requirements during pregnancy. Bone Res. 2017 Aug 29; 5:17030.

3. Wei S.Q. Vitamin D and pregnancy outcomes. Curr Opin Obstet Gynecol. 2014 Dec; 26(6):438-47.

4. Harvey N.C., Holroyd C., Ntani G., Javaid K., Cooper P., Moon R., Cole Z., Tinati T., Godfrey K., Dennison E., Bishop N.J., Baird J., 
Cooper C. Vitamin D supplementation in pregnancy: A systematic review. Health Technol Assess. 2014 Jul; 18(45):1-190.

5. Amegah A.K., Klevor M.K., Wagner C.L. Maternal vitamin D insufficiency and risk of adverse pregnancy and birth outcomes: A systematic review and meta-analysis of longitudinal studies. PLoS One. 2017 Mar 17; 12(3):e0173605.

6. Adamova Z., Ozkan S., Khalil R.A. Vascular and cellular calcium in normal and hypertensive pregnancy. Curr Clin Pharmacol. 2009 Sep; 4(3):172-90.

7. Hornsby E., Pfeffer P.E., Laranjo N., Cruikshank W., Tuzova M., Litonjua A.A., Weiss S.T., Carey V.J., O'Connor G.,

Hawrylowicz C. Vitamin D supplementation during pregnancy: Effect on the neonatal immune system in a randomized controlled trial. J Allergy Clin Immunol. 2018 Jan; 141(1):269-278.e1.

8. Chen Y., Zhu B., Wu X., Li S., Tao F. Association between maternal vitamin $\mathrm{D}$ deficiency and small for gestational age: Evidence from a meta-analysis of prospective cohort studies. BMJ Open. 2017 Aug 27; 7(8):e016404.

9. Hadji P., Boekhoff J., Hahn M., Hellmeyer L., Hars O., Kyvernitakis I. Pregnancy-associated osteoporosis: A case-control study. Osteoporos Int. 2017 Apr; 28(4):1393-1399.

10. Carsote M., Valea A. Insights of peak bone mass. Acta Medica Transilvanica. 2016; 21(2):82-85. ISSN 2285-7079, ISSN-L 14531968. 